\title{
Magnetohydrodynamic modeling of three Van Allen Probes storms in 2012 and 2013
}

\author{
J. Paral ${ }^{1,3}$, M. K. Hudson ${ }^{1}$, B. T. Kress ${ }^{2}$, M. J. Wiltberger ${ }^{3}$, J. R. Wygant $^{4}$, and H. J. Singer ${ }^{5}$ \\ ${ }^{1}$ Department of Physics and Astronomy, Dartmouth College, Hanover, NH, USA \\ ${ }^{2}$ Cooperative Institute for Research in Environmental Sciences, University of Colorado, Boulder, CO, USA \\ ${ }^{3}$ High Altitude Observatory, NCAR, Boulder, CO, USA \\ ${ }^{4}$ Department of Physics and Astronomy, University of Minnesota, Minneapolis, MN, USA \\ ${ }^{5}$ NOAA Space Weather Prediction Center, Boulder, CO, USA
}

Correspondence to: J. Paral (jparal@gmail.com)

Received: 18 March 2015 - Revised: 4 August 2015 - Accepted: 9 August 2015 - Published: 20 August 2015

\begin{abstract}
Coronal mass ejection (CME)-shock compression of the dayside magnetopause has been observed to cause both prompt enhancement of radiation belt electron flux due to inward radial transport of electrons conserving their first adiabatic invariant and prompt losses which at times entirely eliminate the outer zone. Recent numerical studies suggest that enhanced ultra-low frequency (ULF) wave activity is necessary to explain electron losses deeper inside the magnetosphere than magnetopause incursion following CME-shock arrival. A combination of radial transport and magnetopause shadowing can account for losses observed at radial distances into $L=4.5$, well within the computed magnetopause location. We compare ULF wave power from the Electric Field and Waves (EFW) electric field instrument on the Van Allen Probes for the 8 October 2013 storm with ULF wave power simulated using the Lyon-FedderMobarry (LFM) global magnetohydrodynamic (MHD) magnetospheric simulation code coupled to the Rice Convection Model (RCM). Two other storms with strong magnetopause compression, 8-9 October 2012 and 17-18 March 2013, are also examined. We show that the global MHD model captures the azimuthal magnetosonic impulse propagation speed and amplitude observed by the Van Allen Probes which is responsible for prompt acceleration at $\mathrm{MeV}$ energies reported for the 8 October 2013 storm. The simulation also captures the ULF wave power in the azimuthal component of the electric field, responsible for acceleration and radial transport of electrons, at frequencies comparable to the electron drift period. This electric field impulse has been shown to explain observations in related studies (Foster et al., 2015) of elec-
\end{abstract}

tron acceleration and drift phase bunching by the Energetic Particle, Composition, and Thermal Plasma Suite (ECT) instrument on the Van Allen Probes.

Keywords. Magnetospheric physics (electric fields; MHD waves and instabilities; solar wind-magnetosphere interactions)

\section{Introduction}

The twin Van Allen Probes spacecraft, launched on $30 \mathrm{Au}-$ gust 2012, are providing dual-spacecraft measurements of radiation belt electron response to changing electric and magnetic fields, particularly dramatic for coronal mass ejection (CME)-shock-driven storms. Inward motion of the magnetopause causes loss on the timescale of magnetopause compression (Baker et al., 2012; Hudson et al., 2014a) evident in loss of electrons at high $L$ values. The Van Allen Probes measure electron and ion populations across a broad range of energies with complementary electric and magnetic field measurements designed to answer fundamental questions about the balance between source and loss processes affecting the most energetic (multi-MeV) outer-zone electrons in particular (Mauk et al., 2013). An issue is the balance between acceleration by radial transport vs. local wave processes (Hudson et al., 2008; Thorne, 2010) and the balance between loss to the magnetopause and atmosphere (Millan and Thorne, 2007). Both dramatic dropouts (Turner et al., 2014) and dramatic increase in the flux of $\mathrm{MeV}$ electrons have been reported (Reeves et al., 2013). 
CME-driven compression of the dayside magnetopause produced a dropout of $\mathrm{MeV}$ electron flux into the location of the plasmapause along with the formation of a storage ring at multi-MeV energies from the residual outer zone during the first observed storm on 3 September 2012 (Baker et al., 2013). This storage ring persisted for the remainder of September 2012, but was largely decimated by inward motion of the magnetopause when a strong interplanetary shock arrived at Earth on 30 September (Turner et al., 2014). Another CME shock erased the last vestiges of the storage ring on 8 October 2012, attributed to magnetopause shadowing (Hudson, 2013), followed by a very rapid acceleration event seen by both Van Allen Probes spacecraft on 9 October (Reeves et al., 2013), attributed to local acceleration by gyroresonance with whistler-mode chorus waves (Thorne et al., 2013).

The role of ULF waves in radial transport has been suggested by long periods of gradual inward motion at fixed energies as occurred in the first half of March 2013 (Baker et al., 2014; Li et al., 2014b). This behavior was interrupted by prompt loss of the outer zone on 17 March following the arrival of another strong CME shock, which produced a $D_{\text {st }}$ of $-132 \mathrm{nT}$ and subsequent local acceleration comparable to the 8-9 October 2012 storm ( $\mathrm{Li}$ et al., 2014a). The role of ULF waves during this and the preceding 8-9 October 2012 storm event, which were examined globally in Hudson et al. (2014a, b), is examined in more detail here, including further analysis of the magnetosonic impulse which accompanies magnetosonic compression.

In this paper we present magnetohydrodynamic (MHD) simulations for the 8-9 October 2012 and 17-18 March 2013 CME-shock-driven storms along with a third 8-9 October 2013 storm which has received considerable attention because of the observation of prompt acceleration on an electron drift timescale in addition to the characteristic prompt loss due to magnetopause compression (Foster et al., 2015). A detailed comparison of simulated and measured electric field data is available for this storm due to precession of the spacecraft orbit to the dayside by October 2013. Foster et al. (2015) estimated, based on observations from the Van Allen Probes, that the moderate azimuthal electric field impulse of $\sim 10 \mathrm{mV} \mathrm{m}^{-1}$ observed by the Electric Field and Waves (EFW) instrument in response to dayside compression, with spatial extent over the entire dayside magnetosphere, was responsible for selective acceleration of $1.5-4.5 \mathrm{MeV}$ electrons by $400 \mathrm{keV}$ in the radial region of $L^{*} \sim 3.5-4.0$.

\section{Measurements}

The orbital period of the Van Allen Probes is about $9 \mathrm{~h}$ with apogee at 5.8 $R_{\mathrm{E}}$ (Mauk et al., 2013). The acquired electron and ion particle distribution functions with unprecedented energy, pitch angle and temporal resolution in the range from a few eV to $\mathrm{MeV}$ are measured by three different instruments in the Energetic Particle, Composition, and Thermal
Plasma Suite (ECT) (Spence et al., 2013). The Electric Field and Waves (EFW) instrument (Wygant et al., 2013) on the Van Allen Probes is composed of two booms with spherical probes and tip-to-tip separation of $100 \mathrm{~m}$ in the spin plane. The third component along the spin axis is measured by two spherical sensors separated by $15 \mathrm{~m}$. The instrument works nominally in a survey mode of 32 samples per second but can use two burst memories with sampling rate of 512 or 16000 samples per second. An accurate measurement of the electric field is particularly important in determining the effects of particle acceleration (Foster et al., 2015), the losses to both the atmosphere and magnetopause (Hudson et al., 2014a), and also microphysical phenomena like double layers (Mozer et al., 2014). Thanks to the orbit design of the two spacecraft, we receive a current picture of the radiation belts with at least $4.5 \mathrm{~h}$ resolution (half orbital period), and are able to separate temporal from spatial variations on a shorter timescale using the spatial separation of the two spacecraft. This time resolution may be sufficient for changes of particle fluxes, but to study changes in the electric and magnetic field configuration even on MHD timescales, we require higher temporal and spatial coverage than is possible to achieve with two-point measurements.

In this paper, we selected three events associated with coronal mass ejections (CMEs) from 2012 and 2013 to simulate using a global MHD model driven by upstream solar wind measurements. We focus on ultra-low frequency (ULF) wave activity of the azimuthal component of the electric field which affects radial transport of electrons. To establish a baseline, we choose the 8 October 2013 event and compare the electric field observations with the results of the numerical model. This event, well measured by the Van Allen Probes, is a good example of the magnetosphere response to the induced electric field associated with a CME-shock impact.

GOES 13 and 15 geosynchronous satellites and the CARISMA magnetometer chain were also on the dayside for the 8 October 2013 CME-shock arrival, making it possible to directly compare the magnetic field compression and azimuthal impulse propagation speed seen in those measurements with the Van Allen Probes data and MHD simulations described next.

\section{Computational methods}

\subsection{MHD model}

In this study, we use the global Lyon-FedderMobarry (LFM) MHD model described in detail by Lyon et al. (2004). LFM is coupled with two other models which improve description of physical processes in the inner magnetosphere. The Rice Convection Model (RCM) provides a representation of ring current particle populations including drift physics (Toffoletto et al., 2003; Pembroke et al., 2012) and the Magnetosphere-Ionosphere 
Coupler/Solver (MIX) model provides an inner boundary at $2 R_{\mathrm{E}}$ (Merkin and Lyon, 2010).

LFM is a three-dimensional magnetospheric model which solves ideal single-fluid magnetohydrodynamic (MHD) equations on an irregular grid stretched so it conforms with topology of the Earth's dipole as distorted by the solar wind. The grid has higher resolution in the inner magnetosphere of about $0.25 R_{\mathrm{E}}$. Internally, LFM uses the solar magnetic (SM) coordinate system where the $z$ axis coincides with the geomagnetic dipole axis and the $x-z$ plane contains the SunEarth line. The model is driven by solar wind conditions upstream of the simulation box imposed on a boundary located at $x_{\mathrm{SM}}=30 R_{\mathrm{E}}$ and is rotated appropriately to model diurnal variations. The solar wind data are derived from either Advanced Composition Explorer (ACE) and Wind spacecraft located near the L1 Lagrangian point or the ARTEMIS spacecraft orbiting the Moon. The input data are interpolated on a regular time grid with cadence of either 60 or $6 \mathrm{~s}$ when ARTEMIS data are used.

RCM assumes a Maxwellian distribution function for both electrons and ions decomposed in energy into 28 fluid channels for electrons and 62 channels for ions (Toffoletto et al., 2003). The flux tube averaged density, temperature and magnetic field are provided by LFM, while RCM provides the sound speed which is bled into LFM with a characteristic exchange time and a coupling ratio. RCM solves a 2-D bounce-averaged advection equation separately for each energy channel in the spatial region where the flux tube averaged $\beta \leq 1$. Bounce averaging assumes spherical symmetry between northern and southern ionospheres, which is satisfied due to the spatial restriction described above. The two models are coupled with 1 min cadence. A static Gallagher plasmasphere (Gallagher et al., 2000) for $K p=5$ and constant azimuthal profile is added to the output during the RCM to LFM coupling step. The cold plasma density has a profile in the radial direction with constant plasmapause location set at $4.5 R_{\mathrm{E}}$. RCM has an option of incorporating dipole tilt which is enabled for all runs presented in this paper. Twoway coupling between LFM and RCM is discussed in Pembroke et al. (2012).

The Magnetosphere-Ionosphere Coupler/Solver (MIX) model solves for the electric potential $\Phi$ from field aligned currents and conductivity using a 2-D continuity equation:

$\nabla_{\perp} \cdot \bar{\Sigma} \cdot \nabla_{\perp} \Phi=J_{\|} \cos \alpha$,

where $\bar{\Sigma}$ is the height-integrated conductance tensor modulated by $10.7 \mathrm{~cm}$ solar radio flux, $\Phi$ is the electric potential and $J_{\|}$are field-aligned currents mapped from the inner boundary of LFM along the dipole field onto the grid of MIX. The angle $\alpha$ is between radial and local magnetic field on the grid. Equation 1 is solved on a 2-D spherical grid. The implementation details are discussed in Wiltberger et al. (2004). Throughout this paper we will refer to all three models coupled together as LFM-RCM.

\subsection{Solar wind conditions}

LFM-RCM requires proton density, velocity, magnetic field and sound speed of the solar wind as input parameters, together with angle between magnetic and geographic pole to account for diurnal variations. All solar wind input parameters are interpolated on a $1 \mathrm{~min}$ time grid or $6 \mathrm{~s}$ in the case of ARTEMIS input. We use three different sources of solar wind data based on availability.

ACE and the Global Geospace Science Wind spacecraft are strategically located in orbit about the L1 Lagrangian point to provide near-real-time space weather measurements upstream of Earth. Solar wind measurements are then propagated to the Earth's bow shock nose and provided by NASA's OMNIWeb service. ACE was launched on 25 August 1997 and its $X_{\mathrm{GSE}}$ ranges between 220 and $250 R_{\mathrm{E}}$. Wind was launched on 1 November 1994 and still provides solar wind data, but from mid-2004 its orbit has an excursion in $Y_{\mathrm{GSE}}$ $\pm 100 R_{\mathrm{E}}$.

ARTEMIS are two spacecraft orbiting the Moon which were separated from the THEMIS (B and C spacecraft) mission (Angelopoulos, 2008, 2010). ARTEMIS data were smoothed by using a boxcar average algorithm of $40 \mathrm{~s}$ window size. The original data were sampled at about $4 \mathrm{~s}$. Afterwards, data were interpolated on a $6 \mathrm{~s}$ time grid to preserve a steep jump in solar wind parameters.

\subsection{Power analysis}

ULF wave power is analyzed in the SM equatorial plane, as this plane approximates the $B_{\min }$ surface. We plotted the $B_{\text {min }}$ surface in the LFM fields in SM coordinates using a field line tracer and found that this surface is within $6^{\circ}$ of the SM equatorial plane, valid within the inner magnetosphere. The fields from the MHD simulation are saved every $30 \mathrm{~s}$, which corresponds to a Nyquist frequency of $\sim 16 \mathrm{mHz}$.

MHD models resolve two coupled wave modes which produce $E_{\phi}$, which in turn provides a mechanism responsible for radial particle transport. First, a fast magnetosonic wave mode can be launched from sudden compression of the dayside portion of the magnetosphere after shock arrival (see Araki et al., 1997, for analysis of the 24 March 1991 storm sudden commencement (SSC) using satellite and ground magnetometer measurements). This wave propagates isotropically in low $\beta$ plasmas from a source region on the magnetopause. Due to the dipolar topology of the $B$ field and inhomogeneities in the plasma, the magnetosphere focuses the waves into the equatorial plane region as they propagate (Kress et al., 2007). Global ULF wave oscillations are another source of $E_{\phi}$. The global oscillations of shear Alfvén waves are excited by coupling with fast magnetosonic waves through field line resonance (see the review of Hughes, 1994).

To analyze ULF wave activity we apply a Hanning window of length of $30 \mathrm{~min}$ and $50 \%$ overlap (i.e., $15 \mathrm{~min}$ ) to 
electric field detrended by a polynomial of order 3 before data are processed by fast Fourier transform (FFT). The electric field is measured along the Sun-Earth line in analysis of the simulated spatial domain.

Figures. 1, 2 and 3, which represent ULF wave power analysis, all follow the same format. Panel (a) is ULF wave power integrated over the range 0 to $16 \mathrm{mHz}$ as a function of ra-

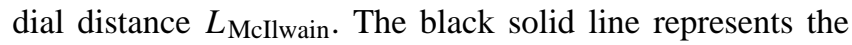
location of the magnetopause and the dashed line is the geostationary radial distance at $6.6 R_{\mathrm{E}}$. Magnetopause location is calculated from the maximal gradient of the number density sampled along the Sun-Earth line. As shown by Hudson et al. (2014a), LFM-RCM predicts the magnetopause location well with respect to a widely used empirical model of Shue et al. (1998). Panel (b) is mean ULF wave power between $L=4$ and $L_{\mathrm{mp}}-1$, where $L_{\mathrm{mp}}$ is the magnetopause location. Panels (c-e) represent solar wind conditions: proton number density, $B_{\mathrm{Z}}$ component of the magnetic field and solar wind dynamic pressure, respectively.

\section{Simulated events}

We present three numerical runs for selected storms of 2012 and 2013 during the primary phase of the Van Allen Probes mission. All three events were weak CME shocks, and are summarized in Table 1. None of the storms was accompanied by a drop of the $D_{\text {st }}$ index below $-150 \mathrm{nT}$. We calculated the peak azimuthal electric field $E_{\phi}$ at geostationary radial distance of $6.6 R_{\mathrm{E}}$ from the simulation during the course of each storm, affecting radial transport of electrons. All three storms reached only modest values of $E_{\phi}$ with a maximum of $-24 \mathrm{mV} \mathrm{m}^{-1}$ during the 8 October 2013 storm. Here we report only the maximal value in the westward direction as it is responsible for inward electron transport; however the eastward component reached similar values within a few percent. Similar signatures of a compressional $B_{\mathrm{Z}}$ have been reported in the past for CME-shock-driven storms, first analyzed in detail with electric and magnetic fields measured in situ by the Combined Release and Radiation Effects Satellite (CRRES) for the 24 March 1991 event (Wygant et al., 1994).

All three storms exhibit increased power during the early phase of the shock arrival as seen in Figs. 1, 2 and 3, which penetrates deep into the inner magnetosphere. Enhanced ULF wave power in the heart of the outer radiation belt will further promote outward radial diffusion and thus loss of the particles to the magnetopause in the presence of an inward radial gradient produced by inward motion of the magnetopause (Shprits et al., 2006; Loto'aniu et al., 2010).

\subsection{October 2012}

The double-dip (in $D_{\text {st }}$ ) storm on 8 October 2012 followed month-long observations of a three-belt structure of highenergy (MeV) electrons reported by Baker et al. (2013). The

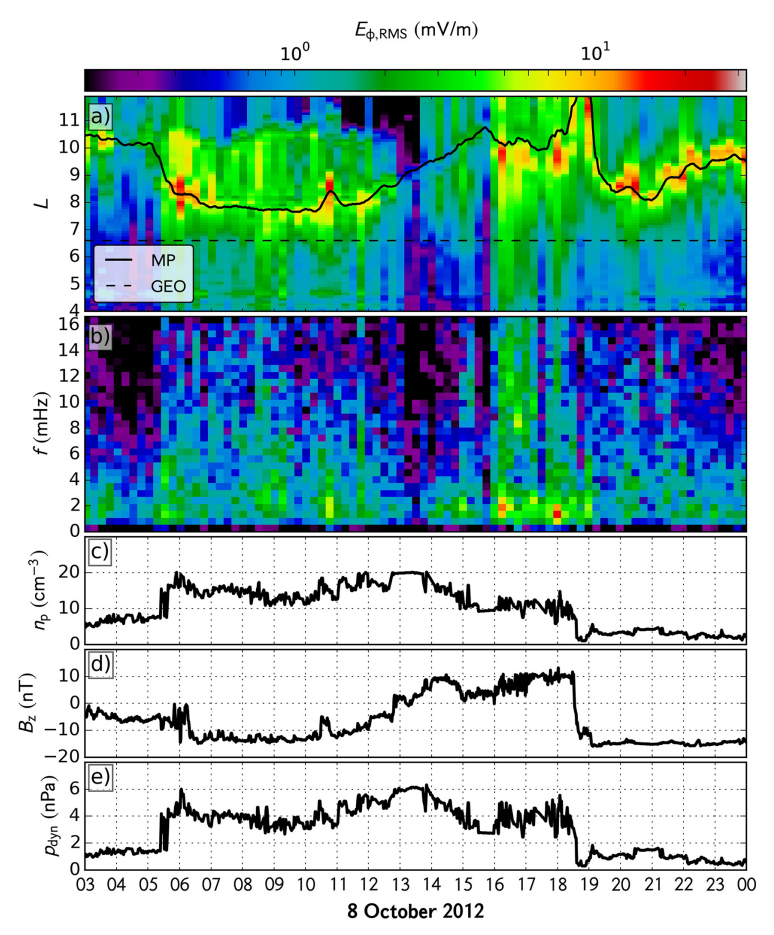

Figure 1. Power spectrum analysis of LFM-RCM run for 8 October 2012 storm along Sun-Earth line of azimuthal component of electric field. Panel (a) is ULF power between 0 and $16 \mathrm{mHz}$ as a function of radial distance in SM coordinate system with magnetopause (solid line) and geostationary (dashed line) location overplotted. Panel (b) is mean ULF wave power from $L=4$ to just inside the magnetopause as a function of frequency. Panels (c-d) are corresponding solar wind parameters used as upstream boundary conditions for LFM-RCM simulation. This figure along with Figs. 2 and 3 reanalyze the data shown in Hudson et al. (2014b) to exclude power in panel (b) which is dominated by magnetopause fluctuations; thus only power inside the black curve in (a) minus $1 R_{\mathrm{E}}$ is plotted in (b).

event preceded sudden energization of the relativistic electrons on 9 October (Reeves et al., 2013). A first dip in $D_{\text {st }}$ of $-105 \mathrm{nT}$ was recorded at the time of increased solar wind dynamic pressure of $6.8 \mathrm{nPa}$. A dip in solar wind pressure and an extended period $\sim 14 \mathrm{~h}$ of southward interplanetary magnetic field (IMF) $B_{\mathrm{Z}}$ was correlated with a sudden increase in the relativistic electron population deep inside geosynchronous orbit. Hudson et al. (2014a) analyzed the 8 October 2012 event in detail using MHD simulations combined with a 3-D particle-tracing technique (Kress et al., 2007) to study the electron loss mechanism due to magnetopause shadowing. The energy and pitch angle dependence of the losses analyzed reveal a clear signature of magnetopause loss at higher $L$ on 8 October observed by the Van Allen Probes ECT instrument. The losses at low $L$ due to high-frequency waves (EMIC and whistler) which produce scattering into the atmosphere were not included in the analysis because the MHD model was used to provide the fields. The long pe- 
Table 1. Event summary: peak $E_{\phi}$ in westward direction measured at GEO.

\begin{tabular}{ll|ccc} 
& & 8 Oct 2012 & 17 Mar 2013 & 8 Oct 2013 \\
\hline \multicolumn{2}{l|}{\begin{tabular}{l|ccc} 
Start of simulation \\
End of simulation
\end{tabular}} & 7 Oct 00:00 UT & 16 Mar 18:00 UT & 8 Oct 08:00 UT \\
& 8 Oct 24:00 UT & 17 Mar 24:00 UT & 9 Oct 18:00 UT \\
\hline $\min \left(D_{\mathrm{st}}\right)$ & $(\mathrm{nT})$ & -105 & -132 & -65 \\
$\min \left(E_{\phi, \mathrm{GEO}}\right)$ & $\left(\mathrm{mV} \mathrm{m}^{-1}\right)$ & -4 & -20 & -24 \\
$\max \left(p_{\text {dyn }}\right)$ & $(\mathrm{nPa})$ & 6.8 & 19.8 & 26.9 \\
$\min \left(L_{\mathrm{MP}}\right)$ & $\left(R_{\mathrm{E}}\right)$ & 7.5 & 6.0 & 5.6 \\
\hline \multicolumn{2}{l}{ Source of solar wind } & Wind & ACE + Wind & ARTEMIS
\end{tabular}

riod of southward IMF $B_{\mathrm{Z}}$ resulted in enhanced convection and plasma sheet electron injection modeled by Kress et al. (2014), providing both an anisotropic population of electrons which generate chorus waves on the dawn side of the magnetosphere and a population of hundreds of $\mathrm{keV}$ electrons inside geosynchronous orbit which may be heated locally by the chorus to $\mathrm{MeV}$ energies (Thorne et al., 2013).

Figure 1 presents ULF wave activity during the 8 October 2012 event. Solar wind conditions derived from the Wind spacecraft were time-shifted to $1 \mathrm{AU}$ to study the properties and intensity of ULF waves inside of the magnetopause location. The initial shock arrival at 05:20 UT launched a pulse propagating through the magnetosphere which generated ULF wave power in the frequency range 1 to $5 \mathrm{mHz}$. Wave power in this frequency range is present throughout the entire simulated event. Note that the plasmapause is static in our simulations. The $K_{\mathrm{p}}=5$ (Gallagher et al., 2000) plasmasphere is incorporated into MHD density. The low level of power at $7-15 \mathrm{mHz}$ does not resonate with electrons below $6 \mathrm{MeV}$ outside of $L=5$ (Fig. 3 of Foster et al., 2015, for ULF wave resonance with electron drift period), assuming an $m=1$ azimuthal mode structure is dominant, characteristic of CME-shock events (Elkington et al., 2012). An analysis of azimuthal mode number structure shows dominance of $m=1$ and 2 for this type of shock compression event (Elkington et al., 2004). At this time, power is centered at $1.5 \mathrm{mHz}$, with an amplitude of $E_{\phi}$, RMS more than $11 \mathrm{mV} \mathrm{m}^{-1}$. Note that panel (b) of Fig. 1 represents only power inside of the magnetopause minus $1 R_{\mathrm{E}}$.

\subsection{March 2013}

On 17 March 2013, a long gradual diffusion of MeV electrons that began on 1 March 2013 with a solar wind highspeed stream was interrupted by a shock arrival (Baker et al., 2014) which was produced by a M1.1-class X-ray flare and subsequent CME. A sudden loss of outer-zone relativistic electrons can be accounted for by wave activity and inward radial motion of the magnetopause below geosynchronous orbit (Li et al., 2014b). The simulated azimuthal electric field at geostationary orbit peaked at $20 \mathrm{mV} \mathrm{m}^{-1}$ during the course of $15 \mathrm{~h}$ of increased solar wind dynamic pressure followed by

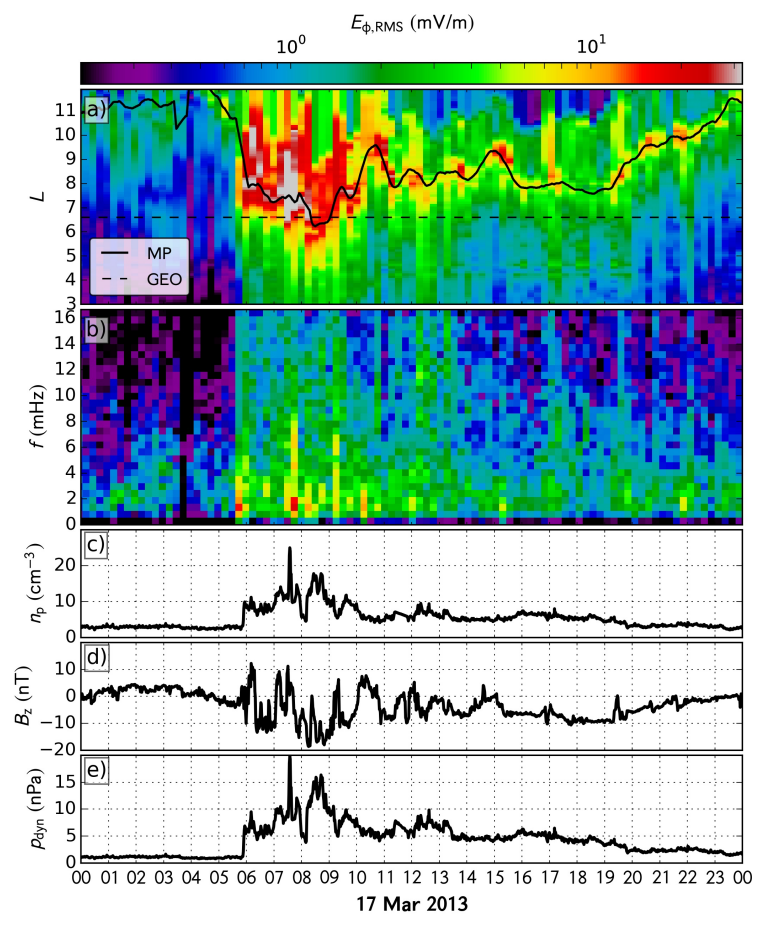

Figure 2. Power spectrum analysis for 17 March 2013 storm. The figure follows the same format as Fig. 1.

rapid energization of electrons at about 18:00 UT observed by the Van Allen Probes (Baker et al., 2014; Foster et al., 2014; Li et al., 2014a).

The upstream solar wind conditions of the LFM-RCM simulation are derived from ACE. Because the ACE data set is incomplete for the given time period, we filled data gaps using the Wind Solar Wind Experiment (SWE) measurements centered on the observation of the shock arrival and downloaded from OMNIWeb. This corresponds to a time shift of ACE observations by $13 \mathrm{~min}$ and $12 \mathrm{~s}$ due to the relative spacecraft location. Wind data are used to fill four gaps in the ACE data set by properly scaling variables to match ACE data during the time range of overlap to account for limited cross calibration of the instruments. 
The 17 March storm was the strongest of the three studied in response of the ring current, with minimum $D_{\text {st }}$ of $-132 \mathrm{nT}$ at 21:00 UT compared to the other two events studied here. Around 07:30 UT the solar wind dynamic pressure reached its maximum of $19.8 \mathrm{nPa}$ and compressed the simulated magnetopause into $L_{\text {Mcllwain }}=6.0$. During this time, the azimuthal electric field derived from the simulation at geosynchronous orbit peaked at $-20 \mathrm{mV} \mathrm{m}^{-1}$.

Figure 2 shows that during the strongest solar wind driving at 07:00 and 08:00 UT, the root mean square of the azimuthal electric field was greater than $11 \mathrm{mV} \mathrm{m}^{-1}$ for frequencies below $4 \mathrm{mHz}$. A similar response was seen during the initial shock arrival at about 05:50 UT. Note that ULF wave power integrated over frequency (and $L$ ) is more intense for the 17 March 2013 event than for that of 8 October 2012.

\subsection{October 2013}

The solar wind input for this simulation uses a combination of data from the Moon-orbiting ARTEMIS P1 and P2 spacecraft (Angelopoulos, 2010). The shock is supercritical with a relatively large magnetic field compression ratio of about $r=4$ (ratio of upstream to downstream magnetic field). The width of the shock ramp is also unusual and lasted only 3-4 s as measured in the solar wind. This fast transition in plasma parameters is basically a delta function on the timescale required by MHD input and is unique relative to other events studied in this paper. Strong constant driving persisted for almost $2 \mathrm{~h}$ and the magnetopause was compressed within geosynchronous orbit for an extended period of time of more than $1.5 \mathrm{~h}$ seen both in the simulation results and GOES magnetometer data shown in Fig. 4b.

Here we present the Van Allen Probes electric field observations to illustrate differences and similarities between our simulations and in situ observations. As seen in Fig. 4, the Van Allen Probes proceeded from the dayside towards dusk at apogee, which was a favorable configuration for the comparison of the observations with the MHD numerical model showing magnetopause compression. Figure 5 shows electric field measurement from the EFW electric field instrument onboard the Van Allen Probes spacecraft (Wygant et al., 2013; Hudson et al., 2014b). The $E_{\mathrm{y}}$ component in the modified geocentric solar ecliptic coordinate system (mGSE) which is in the spin plane of the spacecraft is presented. Note that the spin axis is always aligned within $15^{\circ}$ of the SunEarth line (Mauk et al., 2013). The spacecraft were located between 13:00 and 18:00 MLT in the heart of the electron outer zone during the event. At the time of the shock impact, the spacecraft separation and timing of the observations yield an $E_{\mathrm{y}}$ impulse speed in the inner magnetosphere of $850 \mathrm{~km} \mathrm{~s}^{-1}$ (Foster et al., 2015). A similar analysis of the simulation results yields an azimuthal propagation speed of $700 \pm 200 \mathrm{~km} \mathrm{~s}^{-1}$. A significant error is caused by output cadence saved from LFM, every $10 \mathrm{~s}$. A confirmation of the impulse propagation speed is observation by GOES 13 and

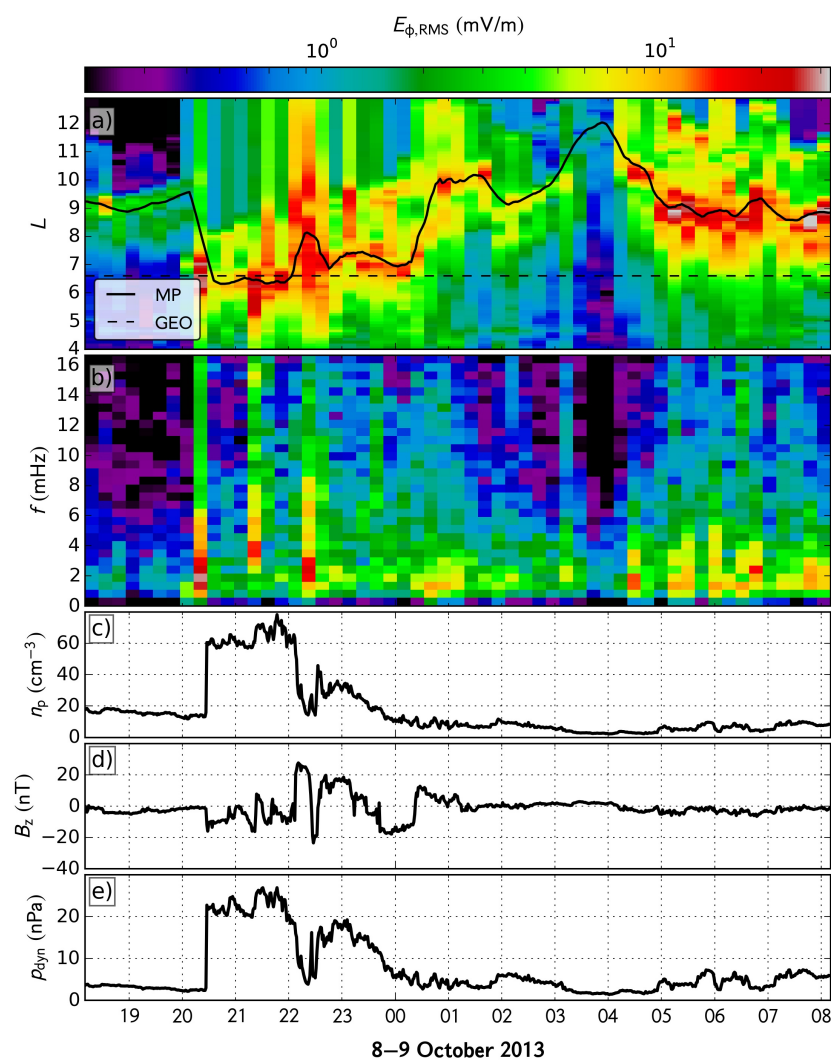

Figure 3. Power spectrum analysis for 8 October 2013 storm. The figure follows the same format as Fig. 1.

15 satellites. Figure $4 \mathrm{~b}$ represents magnetic field measurements of $|B|$. The time difference and spatial separation of the signals yields propagation speed of $978 \mathrm{~km} \mathrm{~s}^{-1}$, which is higher than the speed determined from EFW data from the Van Allen Probes, which were located in a region of lower magnetosonic speed in the simulation (Fig. 4a).

Figure 5 presents $E_{\mathrm{y}, \mathrm{mGSE}}$ (black) and Poynting flux $S_{\mathrm{x}, \mathrm{mGSE}}$ (red) components from both spacecraft in panels (a) and (c). Note that $E_{\mathrm{y}}$ corresponds to the azimuthal or radial direction when spacecraft are located on the dayside or dusk, respectively. Both quantities are band-pass-filtered between 1 and $6 \mathrm{mHz}$. Initially, bipolar oscillations of the $x$ component of the Poynting flux indicate a standing wave, while subsequently, when the spacecraft moves towards apogee and dusk, negative $x$ Poynting flux may be interpreted as an antisunward-propagating wave. The initial impulse at 20:20 UT caused prompt energization by radial transport of multi-MeV electrons as described by Foster et al. (2015), while inward motion of the magnetopause depleted the outerzone radiation belt of relativistic electrons (Hudson et al., 2014b). Relatively high amplitude monochromatic oscillations in the ULF range at later times are ideal for comparing observations with LFM-RCM predicted wave activity.

Figures $5 \mathrm{~b}$ and $5 \mathrm{~d}$ show power spectral density (PSD) in $E_{\mathrm{y}}$ from the same data set as panels (a) and (c). See accom- 

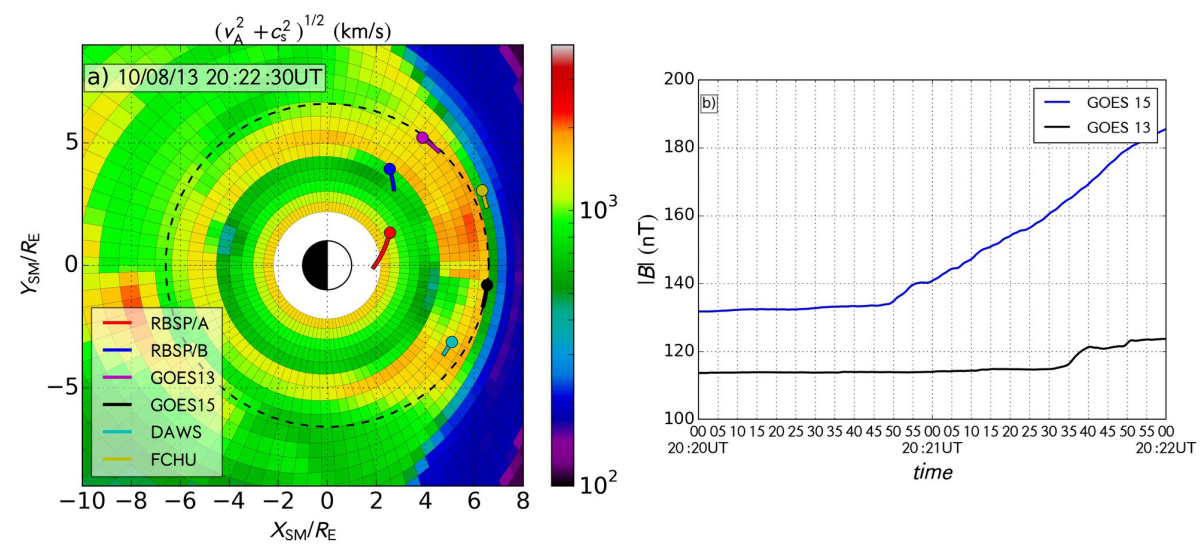

Figure 4. The color-coded logarithm of magnetosonic speed (panel a) extracted from LFM simulation at SM equatorial plane at the time of shock arrival. Location of the Van Allen Probes, GOES 13 and 15 satellites, and CARISMA's Dawson City and Fort Churchill magnetometer stations during 8 October 2013 at 20:22:30 UT in the SM coordinate system. Panel (b) shows magnetic field measurement by GOES satellites around the shock arrival time. Timing analysis yields a propagation speed of $978 \mathrm{~km} \mathrm{~s}^{-1}$ from GOES 15 to GOES 13 .

panying Appendix A and Fig. A1, which, instead of a color spectrogram, presents PSD at four successive times at spacecraft A and B: 21:31, 21:46, 22:01 and 22:16 UT. These times follow shock arrival at 20:22 UT and correspond to times when the wave power is traveling antisunward according to the Poynting flux plotted in panels (a) and (c) of Fig. 5. By these times both spacecraft have advanced duskward from their position at an earlier time of 20:22:30 UT shown in Fig. 4a, where red (blue) symbols indicate the locations of spacecraft A (B).

The $E_{y}$ component is a combination of poloidal- and toroidal-mode power in a non-axisymmetric background magnetic field where the two modes are coupled (Radoski, 1967; see also review by Lanzerotti and Southwood, 1979), and increasingly corresponds to the toroidal mode $\left(E_{\mathrm{r}}\right.$, $B_{\phi}$ ) as the spacecraft move to the dusk side. Thus power shown can be interpreted in terms of excitation of toroidalmode field line resonances by antisunward-propagating magnetosonic-mode wave power (Hughes, 1994), which continues to be sustained by solar wind pressure oscillations (present in Fig. 3e). Note that power is concentrated at lower frequencies at spacecraft B at the earlier time of 21:31 UT (dark-blue curve of Fig. A1) and higher frequency at spacecraft A when it is at a lower $L$ value, as expected for a fundamental field line resonance wave frequency to which these correspond. See Claudepierre et al. (2010), who performed a controlled experiment with LFM and both monochromatic and broadband prescribed pressure oscillations in the frequency range $0.1-50 \mathrm{mHz}$. They found that odd harmonic fundamental toroidal-mode field line resonance power was excited at successively higher frequencies at lower $L$ values. As spacecraft A moves outward in $L$ in Fig. 4 a, the peak in PSD in Fig. A1a shifts to lower frequency by 22:01 UT (red) with power exceeding that seen at spacecraft B at 21:31 UT in Fig. A1b, when it was already at higher $L$. Both space- craft see approximately the same peak frequency $3.3 \mathrm{mHz}$ by 22:01 UT, with power significantly lower at spacecraft B at that time and reduced further as it passes by 22:16 UT and moves further duskward. Figure 5 shows that the $\mathrm{mHz}$ ULF wave PSD attenuates away from the dayside, particularly clear for spacecraft B in panel (d), which has moved more duskward than A, as has been seen in many studies, with concentration of toroidal-mode power along the flanks of the magnetosphere and compressional in B (poloidal $E_{\phi}$ ) power across the dayside; see, for example, Anderson et al. $(1992 a, b)$ for a review of AMPTE CCE magnetometer measurements, Lessard et al. (1999) for analysis of the local time distribution of AMPTE IRM magnetometer data, Hudson et al. (2004) for CRRES magnetometer, Liu et al. (2009) for THEMIS data, and Engebretson et al. (1998) for ground magnetometer studies. Other LFM CME-shock event studies (Elkington et al., 2002, 2012) have confirmed the predominance of wave power on the dayside in the poloidal $E_{\phi}$ component and discussed the need to include that longitudinal asymmetry in any prescribed ULF wave model of effects on radial transport and acceleration of electrons.

Figure 6 represents the simulated color-coded azimuthal electric field $E_{\phi}$ in the SM equatorial plane shortly after the shock arrival at the magnetosphere at 20:22 UT. Overplotted are the electric field vectors (white) projected onto the SM equatorial plane. The location of the two spacecraft is represented by red (A) and blue (B) circles with $30 \mathrm{~min}$ of the past trajectory represented by a solid line of the same color. The dusk-dawn azimuthal electric field in excess of $10 \mathrm{mV} \mathrm{m}^{-1}$ will effectively transport electrons inward through $E \times B$ drift superimposed on the gradient-curvature drift, thus providing adiabatic heating as first and second invariants are conserved (Foster et al., 2015). An equivalent view is that electrons accelerated by $E_{\phi}$ move radially inward while conserving their first invariant (Li et al., 1993). A short duration of the elec- 


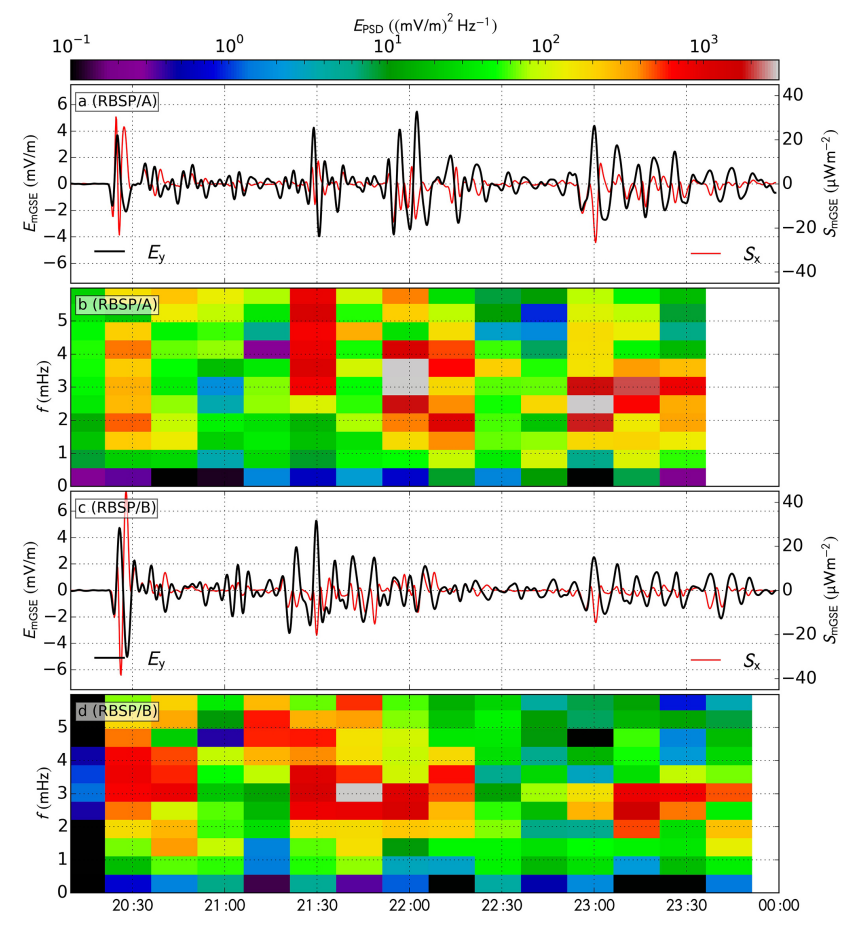

Figure 5. 8 October 2013 event: Poynting flux $S_{\mathrm{xGSM}}$ (red line) and electric field $E_{\mathrm{yGSM}}$ (black line) measurement by the EFW instrument onboard the Van Allen Probe A and B (panels a and c respectively) and corresponding power spectral density computed on $E_{\mathrm{y}}$ in panels (b) and (d), respectively. Note that data are bandpass-filtered between 1 and $6 \mathrm{mHz}$.

tric field pulse will transport only a fraction of the electrons located on the dayside of the magnetosphere, which will lead to drift echoes observed by the Van Allen Probes. Multi-MeV electrons which traverse duskward and stay in drift resonance with azimuthally propagating $E_{\phi}<0$ are most strongly accelerated (Li et al., 1993; Elkington et al., 2002; Kress et al., 2007; Mann et al., 2013)

Figure 6a shows $E_{\phi}$ at the time of shock arrival 20:22:00, while Fig. $6 \mathrm{~b}$ shows $E_{\phi}$ at 20:23:00 UT, after the magnetosonic impulse produced by the dayside compression has propagated toward the nightside. Multi-MeV electrons drifting in resonance with the negative (blue) azimuthal electric field impulse as it propagates eastward from the dayside to nightside experience radial transport and acceleration by $\sim 400 \mathrm{keV}$ in this event, commensurate with the amplitude of $E_{\phi}$ integrated over the electron drift path (Foster et al., 2015). This energization is considerably less than seen in MHD-test particle simulations of the Halloween 2003 CMEshock event where an order of magnitude larger $E_{\phi}$ was produced, using measured solar wind input from a faster, stronger shock (Kress et al., 2007), and for the 24 March 1991 event (Elkington et al., 2002), for which nightside electric field and particle measurements were available from CRRES and $E_{\phi}$ extrapolated to the dayside was found to be $\sim 200 \mathrm{mV} \mathrm{m}^{-1}$ (Wygant et al., 1994). Nonetheless, the dualspacecraft measurements available for the 8 October 2013 event provide a key constraint in determining the consistency of the azimuthal impulse propagation speed between the two spacecraft, separated azimuthally on the dayside at the time of shock arrival as seen in Fig. 6a, and confirmed in the simulations which yield an average $E_{\phi}$ impulse propagation speed of $700 \pm 200 \mathrm{~km} \mathrm{~s}^{-1}$. The GOES 13 and 15 satellites (Fig. 4b) and Canadian magnetometer array CARISMA (Appendix Fig. B1) also confirm very similar propagation speeds of 978 and $780 \mathrm{~km} \mathrm{~s}^{-1}$, respectively, both mapping to higher radial locations in the equatorial plane where the magnetosonic speed is larger (Fig. 4a).

Figure 7a shows the color-coded power spectrum of the $E_{\mathrm{y}, \mathrm{mGSE}}$ electric field component, roughly azimuthal, measured by EFW on Van Allen Probe B (solid black line) extracted along the trajectory between 20:00 and 24:00 UT on 8 October 2013. Panel (b) shows the simulated $E_{\mathrm{y}, \mathrm{SM}}$ electric field in the same format. The bottom four panels $(\mathrm{e}-\mathrm{h})$ show solar wind parameters for the given time period. Panel (b) shows simulated $E_{\mathrm{y}, \mathrm{SM}}$ plotted in the fixed SM coordinate system, while panel (a) is plotted in a coordinate system with $x$ axis pointing along the spacecraft spin axis (Wygant et al., 2013; Hudson et al., 2014a) within $15^{\circ}$ of the Sun-Earth line, and thus is closer to the SM coordinate system of the simulation at the time of shock arrival when the two $y$ components are in relatively close agreement. Both panels (a, measured) and (b, simulated) show similar frequency response to changes in solar wind driving conditions in the lower panels, and similar amplitudes at the time of shock arrival, with larger measured amplitudes by up to a factor of 4 at later times.

Panels (c) and (d) compare the decomposition of simulated $E_{\mathrm{y}, \mathrm{SM}}$ into azimuthal and radial components. The simulated bipolar electric field impulse at 20:22 UT is expected from a compression of the dayside magnetic field following Faraday's law (Li et al., 1993) with a predominantly negative $E_{\mathrm{y}}$ component at the location of the RBSP B spacecraft followed by a smaller damped relaxation oscillation with a $3 \mathrm{~min}(3.3 \mathrm{mHz})$ period. The increase in wave power at 21:25 UT and again at 22:25 UT is associated with subsequent discontinuities in the solar wind and seen as probe B moves from dayside toward the flanks of the magnetosphere (Fig. 4a), where wave power is greater in the toroidal $\left(E_{\mathrm{r}}\right)$ component than the poloidal $E_{\phi}$ component as expected (Fig. 8 of Liu et al., 2009, THEMIS statistical study).

\section{Discussion and summary}

We have presented results from simulations of three CMEshock-driven storms since the launch of the twin Van Allen Probes spacecraft in August 2013. These events were selected because of the dramatic change in radiation belt electron populations that ensued. What they all have in common is compression of the dayside magnetopause and prompt 

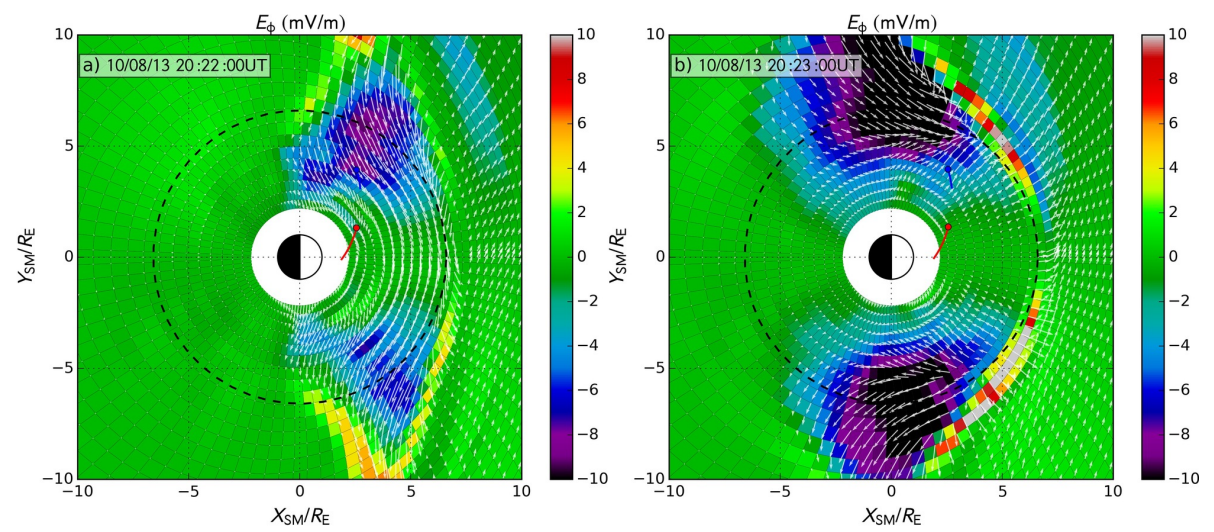

Figure 6. 8 October 2013 event: color-coded $E_{\phi}$ component of electric field in SM equatorial plane shortly after the shock arrival at (a) 20:22 and (b) 20:23 UT, with the electric field vectors overplotted as the white arrows. The locations of Van Allen Probes A (red) and B (blue) are represented by circles with past $30 \mathrm{~min}$ of the trajectory represented by solid line of respective color. Bluer colors, which represent negative electric field, will transport electrons inwards while providing adiabatic heating of particles.

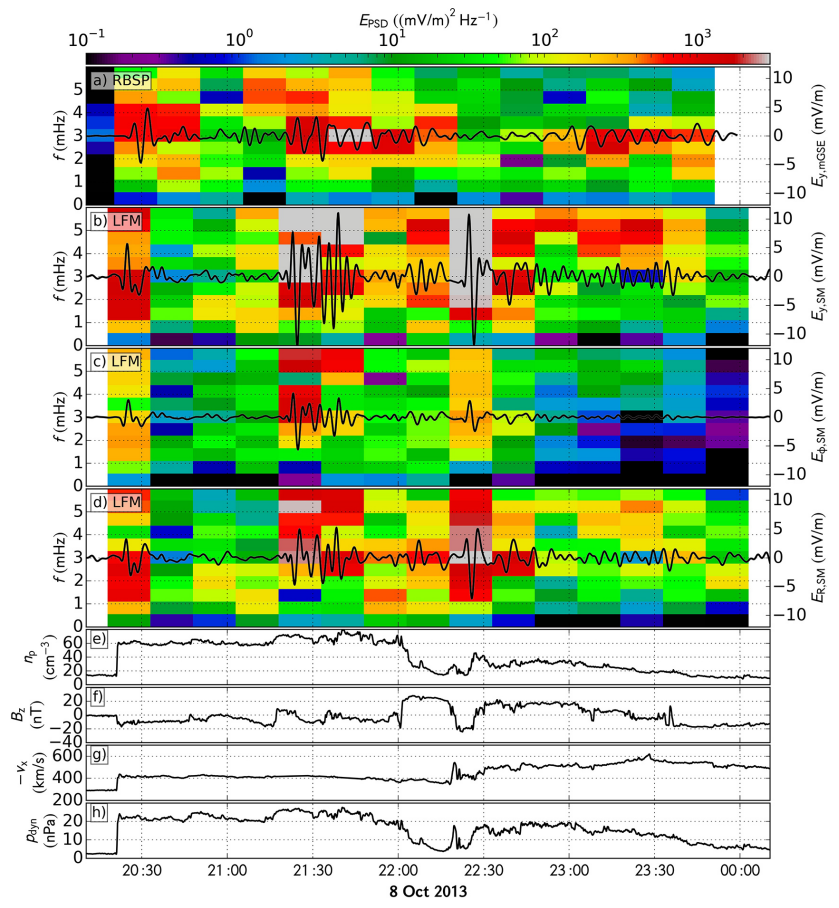

Figure 7. (a) EFW measured in mGSE (modified GSE; see Wygant et al., 2013) at RBSP B and (b) simulated (along RBSP B trajectory) $E_{\mathrm{y}, \mathrm{SM}}$, (c) $E_{\phi, \mathrm{SM}}$ and (d) $E_{\mathrm{R}, \mathrm{SM}}$ for $4 \mathrm{~h}$ interval including the shock impulse arrival at 20:22 UT. The black line in panels (a-d) represents the electric field amplitude indicated on right. Measured solar wind input from ARTEMIS is shown in (e-h).

loss of electrons due to magnetopause shadowing, as has been reported in other studies (Hudson et al., 2014a). The focus of this paper has been the evolution of fields calculated from the simulation model using measured solar wind input from ACE, Wind and ARTEMIS spacecraft. The LFM-RCM model couples the Lyon-Fedder-Mobarry
(LFM) global MHD code with the Rice Convection Model (RCM) of the ring current and includes dipole tilt and a $K p=$ 5 non-evolving embedded plasmasphere for storm time simulations (see Pembroke et al., 2012; Hudson et al., 2014a).

The results capture well the azimuthal electric field impulse that was measured by the EFW instrument on the Van Allen Probes for the 8 October 2013 storm, propagating eastward in the post-noon sector in the direction of electron drift at $\sim 850 \mathrm{~km} \mathrm{~s}^{-1}$ (Foster et al., 2015). Simulation results shown in Fig. 6 yield $700 \pm 200 \mathrm{~km} \mathrm{~s}^{-1}$, which is in good agreement with the Van Allen Probes, GOES and groundbased measurements; the later two data sets were examined for the first time in this paper (see Fig. $4 \mathrm{~b}$ and B1). The simulated $\mathrm{SM} E_{\mathrm{y}} \sim 10 \mathrm{mV} \mathrm{m}^{-1}$ yields an electron energy gain $\sim 380 \mathrm{keV}$ if an electron drifts through a $6 R_{\mathrm{E}}$ azimuthal path length; see for example the snapshot in Fig. 6b, which can account for the prompt energy flux gain observed by the Relativistic Electron Proton Telescope (REPT) component of ECT (Baker et al., 2012) on the Van Allen Probes for this event (Foster et al., 2015). The initial compression yields a negative $E_{\phi}$ across the dayside (frames preceding Fig. 6a), which will account for initial drift phase bunching or drift echoes reported by Foster et al. (2015); however the acceleration continues for those electrons in drift resonance with azimuthal drift velocities comparable to that of the electric field impulse in Fig. 6. The drift-resonant criterion (azimuthal wave velocity equals electron drift velocity) selects an optimal drift-resonant energy $\sim 4 \mathrm{MeV}$ at $L=4$ using a dipole magnetic field approximation to calculate drift period Foster et al. (2015), in line with the drift echo observations detected by the ECT instrument on both spacecraft as they crossed this $L$ value. This is, however, a broad resonance as Hudson et al. (1996) showed for rings of test particle electrons in MHD simulations of the 24 March 1991 event, consistent with the simulated FFT frequency spread evident in Fig. $5 b$ and $d$ at the location of the two spacecraft (A and 
B), as well as the range plotted in Fig. 7. Thus it is not surprising that drift echoes were observed across the energies $2-5.6 \mathrm{MeV}$ of the REPT instrument (Foster et al., 2015) and extended down to lower energies reported for the MagEIS instrument (Blake et al., 2013). This type of prompt acceleration, expected to be more efficient at higher energies and drift velocities which allow electrons to stay in drift resonance with the $E_{\phi}$ impulse plotted in Fig. 6, was shown to be most effective at accelerating electrons above $630 \mathrm{MeV} \mathrm{G}^{-1}$ in phase space density analysis of the 8 October 2013 event (Foster et al., 2015). Prompt drift-resonant acceleration by such an inductive electric field impulse has been reported for other stronger CME-shock compressions of the dayside magnetopause, where much larger amplitude $E_{\phi}$ were inferred from nightside measurements of the March 1991 event by the CRRES satellite (Li et al., 1993) and simulated to explain observations of the Halloween 2003 storm by the low-altitude, polar-orbiting SAMPEX satellite (Looper, 2005; Kress et al., 2007). The results reported here and in companion papers confirm that a much weaker inductive electric field impulse, $\sim 10$ vs. $100 \mathrm{mV} \mathrm{m}^{-1}$, can have a significant effect on electron acceleration during CME-shock-driven storms.
Subsequent stronger ULF wave power at 21:30, 22:00 and post-23:00 UT in Figs. 5, 7 and A1 will continue to affect radial transport of electrons. However, the outer zone was entirely depleted of electrons outside $L=5$ by these later times as the magnetopause moved inside geosynchronous orbit (see Fig. 3, and Hudson et al. (2014b) for corresponding test particle simulations). Electrons were seen to diffuse radially outward beginning with the secondary increase in solar wind dynamic pressure at 21:20 in Fig. 7h, which triggered the second large increase in ULF wave power seen in Figs. 5 and 7. In this case ULF waves can contribute to loss inside the magnetopause due to the reversal of the usual phase space density gradient attributed to magnetopause compression.

Simulating the MHD global response of the magnetosphere to interplanetary drivers such as CME shocks has improved to the point where we can make direct comparisons with dual-spacecraft measurements of large-scale features such as the shock compression inductive electric field impulse seen in Fig. 6. These fields can also be used in studies of longer-timescale behavior such as plasma sheet transport and adiabatic acceleration (Hudson et al., 2012; Kress et al., 2014) as well as radial diffusive acceleration over storm timescales (Elkington et al., 2002, 2004; Fei et al., 2006; Huang et al., 2010; Hudson et al., 2012). 
Appendix A: 8 October 2012: PSD
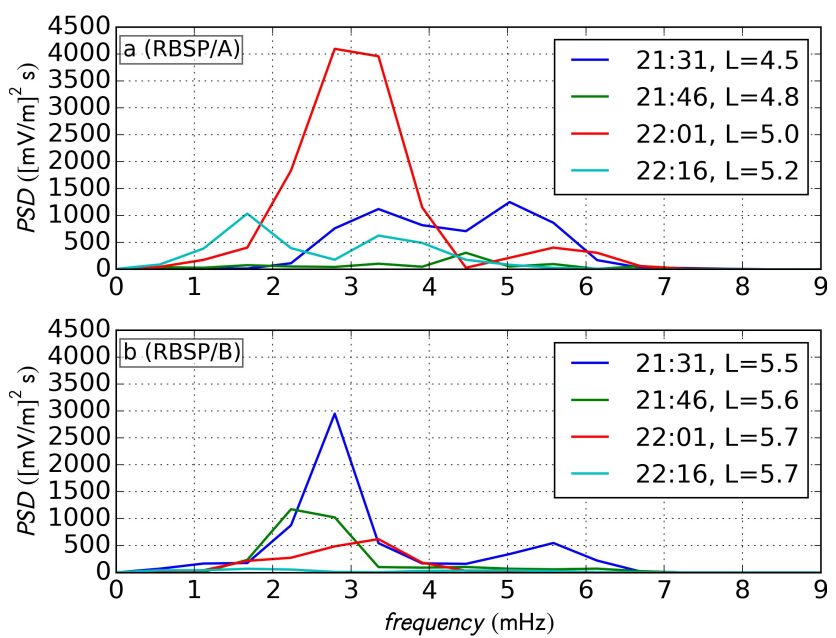

Figure A1. Power spectral density at 21:31, 21:46, 22:01 and 22:16 UT. Note that data are band-pass-filtered between 1 and $6 \mathrm{mHz}$.

Figure A1 follows the same format as Fig. 5 but presents power spectral density at selected times of 21:31, 21:46, 22:01 and 22:16 UT as line plots. The selected times coincide with the shock arrival on 8 October 2013 and subsequent increased wave activity in the frequency range of 1 to $5 \mathrm{mHz}$ which is correlated with changing solar wind conditions plotted in Fig. 7.

\section{Appendix B: 8 October 2012: CARISMA}

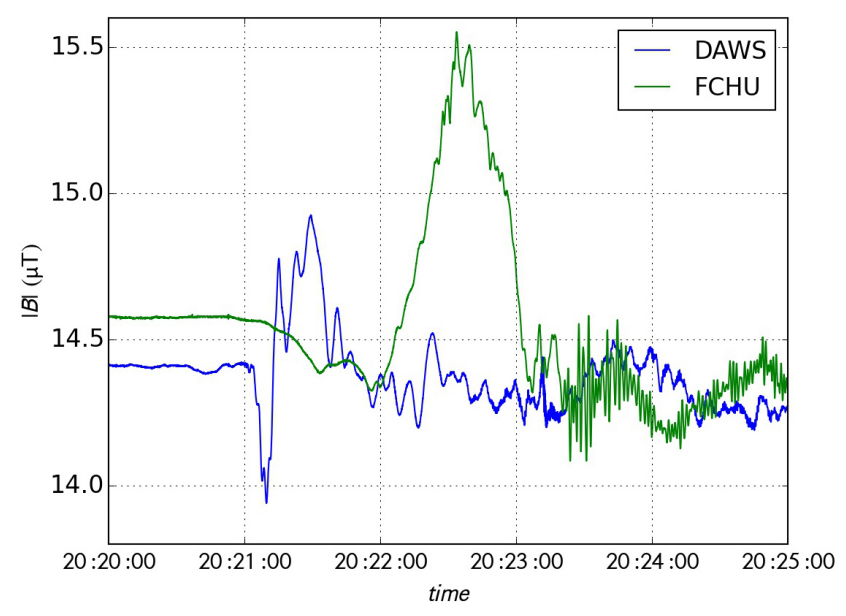

Figure B1. Total magnetic field measured on 8 October 2013 by Dawson City (blue) and Fort Churchill (green) stations from the Canadian magnetometer array CARISMA. The locations of the stations maps approximate to $L=6.1$ and 7.4 $R_{\mathrm{E}}$, respectively, under quiet conditions. The longitudinal separation yields shock impulse propagation speed of $780 \mathrm{~km} \mathrm{~s}^{-1}$.

Figure B1 shows a shock impulse arrival measured by two ground-based magnetometers from the Canadian magnetometer array CARISMA on 8 October 2013. The location of Dawson City (blue) and Fort Churchill (green) stations maps to the dayside at $L=6.1$ and $7.4 R_{\mathrm{E}}$, respectively, under quiet conditions. 
Acknowledgements. This work was supported by JHU/APL under NASA's prime contract NAS5-01072, with work at Dartmouth supported under ECT (967399) subcontract from UNH and EFW (922613) subcontract from UMN. We would like to acknowledge high-performance computing support from Yellowstone provided by NCAR's Computational and Information Systems Laboratory, supported by the National Science Foundation. We thank Drew Turner for providing ARTEMIS solar wind data and Ruth Skoug for discussion of Wind data used to fill ACE data gaps. Van Allen Probe EFW data can be accessed at http://www.space.umn.edu/ rbspefw-data/ and solar wind data (Wind and ACE) are available from http://omniweb.gsfc.nasa.gov/. GOES magnetometer data can be accessed at http://www.ngdc.noaa.gov/ and CARISMA magnetometer data at http://carisma.ca/.

The topical editor B. Mauk thanks two anonymous referees for help in evaluating this paper.

\section{References}

Anderson, B. J., Erlandson, R. E., and Zanetti, L. J.: A Statistical Study of Pc 1-2 Magnetic Pulsations in the Equatorial Magnetosphere, 1. Equatorial Occurrence Distributions, J. Geophys. Res., 97, 3075-3088, doi:10.1029/91JA02706, 1992a.

Anderson, B. J., Erlandson, R. E., and Zanetti, L. J.: A Statistical Study of Pc 1-2 Magnetic Pulsations in the Equatorial Magnetosphere, 2. Wave Properties, J. Geophys. Res., 97, 3089-3101, doi:10.1029/91JA02697, 1992b.

Angelopoulos, V.: The THEMIS Mission, Space Sci. Rev., 141, 534, doi:10.1007/s11214-008-9336-1, 2008.

Angelopoulos, V.: The ARTEMIS Mission, Space Sci. Rev., 165, 3-25, doi:10.1007/s11214-010-9687-2, 2010.

Araki, T., Fujitani, S., Emoto, M., Yumoto, K., Shiokawa, K., Ichinose, T., Luehr, H., Orr, D., Milling, D. K., Singer, H., Rostoker, G., Tsunomura, S., Yamada, Y., and Liu, C. F.: Anomalous sudden commencement on March 24, 1991, J. Geophys. Res., 102, 14075, doi:10.1029/96JA03637, 1997.

Baker, D. N., Kanekal, S. G., Hoxie, V. C., Batiste, S., Bolton, M., Li, X., Elkington, S. R., Monk, S., Reukauf, R., Steg, S., Westfall, J., Belting, C., Bolton, B., Braun, D., Cervelli, B., Hubbell, K., Kien, M., Knappmiller, S., Wade, S., Lamprecht, B., Stevens, K., Wallace, J., Yehle, A., Spence, H. E., and Friedel, R.: The Relativistic Electron-Proton Telescope (REPT) Instrument on Board the Radiation Belt Storm Probes (RBSP) Spacecraft: Characterization of Earth's Radiation Belt High-Energy Particle Populations, Space Sci. Rev., 179, 337-381, doi:10.1007/s11214-0129950-9, 2012.

Baker, D. N., Kanekal, S. G., Hoxie, V. C., Henderson, M. G., Li, X., Spence, H. E., Elkington, S. R., Friedel, R. H. W., Goldstein, J., Hudson, M. K., Reeves, G. D., Thorne, R. M., Kletzing, C. A., and Claudepierre, S. G.: A long-lived relativistic electron storage ring embedded in Earth's outer Van Allen belt, Science, 340, 186-190, doi:10.1126/science.1233518, 2013.

Baker, D. N., Jaynes, A. N., Li, X., Henderson, M. G., Kanekal, S. G., Reeves, G. D., Spence, H. E., Claudepierre, S. G., Fennell, J. F., Hudson, M. K., Thorne, R. M., Foster, J. C., Erickson, P. J., Malaspina, D. M., Wygant, J. R., Boyd, A., Kletzing, C. A., Drozdov, A., and Shprits, Y. Y.: Gradual diffusion and punctuated phase space density enhancements of highly relativistic electrons: Van Allen Probes observations, Geophys. Res. Lett., 41, 1351-1358, doi:10.1002/2013GL058942, 2014.

Blake, J. B., Carranza, P. A., Claudepierre, S. G., Clemmons, J. H., Crain, W. R., Dotan, Y., Fennell, J. F., Fuentes, F. H., Galvan, R. M., George, J. S., Henderson, M. G., Lalic, M., Lin, A. Y., Looper, M. D., Mabry, D. J., Mazur, J. E., McCarthy, B., Nguyen, C. Q., O’Brien, T. P., Perez, M. A., Redding, M. T., Roeder, J. L., Salvaggio, D. J., Sorensen, G. A., Spence, H. E., Yi, S., and Zakrzewski, M. P.: The Magnetic Electron Ion Spectrometer (MagEIS) Instruments Aboard the Radiation Belt Storm Probes (RBSP) Spacecraft, Space Sci. Rev., 179, 383-421, doi:10.1007/s11214-013-9991-8, 2013.

Claudepierre, S. G., Hudson, M. K., Lotko, W., Lyon, J. G., and Denton, R. E.: Solar wind driving of magnetospheric ULF waves: Field line resonances driven by dynamic pressure fluctuations, J. Geophys. Res., 115, A11202, doi:10.1029/2010JA015399, 2010.

Elkington, S. R., Hudson, M. K., Wiltberger, M. J., and Lyon, J. G.: MHD/particle simulations of radiation belt dynamics, J. Atmos. Sol.-Terr. Phy., 64, 607-615, doi:10.1016/S13646826(02)00018-4, 2002.

Elkington, S. R., Wiltberger, M., Chan, A. A., and Baker, D. N.: Physical models of the geospace radiation environment, J. Atmos. Sol.-Terr. Phy., 66, 1371-1387, doi:10.1016/j.jastp.2004.03.023, 2004.

Elkington, S. R., Chan, A. A., and Wiltberger, M.: Global Structure of ULF Waves During the 24-26 September 1998 Geomagnetic Storm, in: Dyn. Earth's Radiat. Belts Inn. Magnetos., edited by Summers, D., Mann, I. R., Baker, D. N., and Schulz, M., 127138, AGU, doi:10.1029/2012GM001348, 2012.

Engebretson, M. J., Glassmeier, K.-H., Stellmacher, M., Hughes, W. J., and Luhr, H.: The dependence of high latitude Pc-5 wave power on solar wind velocity and on the phase of high speed solar wind streams, J. Geophys. Res., 103, 26271, 1998.

Fei, Y., Chan, A. A., Elkington, S. R., and Wiltberger, M. J.: Radial diffusion and MHD particle simulations of relativistic electron transport by ULF waves in the September 1998 storm, J. Geophys. Res., 111, A12209, doi:10.1029/2005JA011211, 2006.

Foster, J. C., Erickson, P. J., Baker, D. N., Claudepierre, S. G., Kletzing, C. A., Kurth, W., Reeves, G. D., Thaller, S. A., Spence, H. E., Shprits, Y. Y., and Wygant, J. R.: Prompt energization of relativistic and highly relativistic electrons during a substorm interval: Van Allen Probes observations, Geophys. Res. Lett., 41, 20-25, doi:10.1002/2013GL058438, 2014.

Foster, J. C., Wygant, J. R., Hudson, M. K., Boyd, A. J., Baker, D. N., Erickson, P. J., and Spence, H. E.: ShockInduced Prompt Relativistic Electron Acceleration In the Inner Magnetosphere, J. Geophys. Res. Space, 120, 1661-1674, doi:10.1002/2014JA020642, 2015.

Gallagher, D. L., Craven, P. D., and Comfort, R. H.: Global core plasma model, J. Geophys. Res., 105, 18819, doi:10.1029/1999JA000241, 2000.

Huang, C.-L., Spence, H. E., Hudson, M. K., and Elkington, S. R.: Modeling radiation belt radial diffusion in ULF wave fields: 2. Estimating rates of radial diffusion using combined MHD and particle codes, J. Geophys. Res., 115, A06216, doi:10.1029/2009JA014918, 2010.

Hudson, M. K.: Space physics: A fast lane in the magnetosphere., Nature, 504, 383-384, doi:10.1038/504383a, 2013. 
Hudson, M. K., Elkington, S. R., Lyon, J. G., Marchenko, V. A., Roth, I., Temerin, M., and Gussenhoven, M. S.: MHD/Particle Simulations of Radiation Belt Formation During a Storm Sudden Commencement, in: Radiat. Belts Model. Stand., edited by Lemaire, J. F., Heynderickx, D., and Baker, D. N., vol. 97 of Geophysical Monograph Series, American Geophysical Union, Washington, D.C., USA, doi:10.1029/GM097p0057, 1996.

Hudson, M. K., Denton, R. E., Lessard, M. R., Miftakhova, E. G., and Anderson, R. R.: A study of Pc-5 ULF oscillations, Ann. Geophys., 22, 289-302, doi:10.5194/angeo-22-289-2004, 2004.

Hudson, M. K., Kress, B. T., Mueller, H. R., Zastrow, J. A., and Bernard Blake, J.: Relationship of the Van Allen radiation belts to solar wind drivers, doi:10.1016/j.jastp.2007.11.003, 2008.

Hudson, M. K., Brito, T., Elkington, S., Kress, B., Li, Z., and Wiltberger, M.: Radiation belt 2D and 3D simulations for CIR-driven storms during Carrington Rotation 2068, J. Atmos. Sol.-Terr. Phy., 83, 51-62, doi:10.1016/j.jastp.2012.03.017, 2012.

Hudson, M. K., Baker, D. N., Goldstein, J., Kress, B. T., Paral, J., Toffoletto, F. R., and Wiltberger, M.: Simulated magnetopause losses and Van Allen Probe flux dropouts, Geophys. Res. Lett., 41, 1113-1118, doi:10.1002/2014GL059222, 2014a.

Hudson, M. K., Kress, B., Paral, J., Baker, D. N., Foster, J. C., Turner, D. L., Wygant, J. R., and Wiltberger, M.: Modeling CME-shock driven storms in 2012-2013: MHDtest particle simulations, J. Geophys. Res., 120, 1168-1181, doi:10.1002/2014JA020833, 2014b.

Hughes, W. J.: Magnetospheric ULF waves: A tutorial with a historical perspective, in: Sol. Wind sources Ultra-Low-Freq. Waves, edited by: Engebretson, M. J., 1-11, Washington, D.C., agu monogr edn., 1994.

Kress, B. T., Hudson, M. K., Looper, M. D., Albert, J., Lyon, J. G., and Goodrich, C. C.: Global MHD test particle simulations of $>10 \mathrm{MeV}$ radiation belt electrons during storm sudden commencement, J. Geophys. Res., 112, A09215, doi:10.1029/2006JA012218, 2007.

Kress, B. T., Hudson, M. K., and Paral, J.: Rebuilding of the Earth's outer electron belt during 8-10 October 2012, Geophys. Res. Lett., 41, 749-754, doi:10.1002/2013GL058588, 2014.

Lanzerotti, L. J. and Southwood, D.: Hydromagnetic Waves, in: Sol. Syst. Plasma Phys., edited by Kennel, C. F., Lanzerotti, L. J., and Parker, E. N., 113-135, Elsevier Science Ltd, New York, USA, 1979.

Lessard, M. R., Hudson, M. K., and Lühr, H.: A statistical study of Pc3-c5 magnetic pulsations observed by the AMPTE/Ion Release Module satellite, J. Geophys. Res., 104, 4523, doi:10.1029/1998JA900116, 1999.

Li, W., Thorne, R. M., Ma, Q., Ni, B., Bortnik, J., Baker, D. N., Spence, H. E., Reeves, G. D., Kanekal, S. G., Green, J. C., Kletzing, C. A., Kurth, W. S., Hospodarsky, G. B., Blake, J. B., Fennell, J. F., and Claudepierre, S. G.: Radiation belt electron acceleration by chorus waves during the 17 March 2013 storm, J. Geophys. Res. Space, 119, 4681-4693, doi:10.1002/2014JA019945, 2014a.

Li, X., Roth, I., Temerin, M., Wygant, J. R., Hudson, M. K., and Blake, J. B.: Simulation of the prompt energization and transport of radiation belt particles during the March 24, 1991 SSC, Geophys. Res. Lett., 20, 2423, doi:10.1029/93GL02701, 1993.

Li, Z., Hudson, M., Jaynes, A., Boyd, A., Malaspina, D., Thaller, S., Wygant, J., and Henderson, M.: Modeling gradual diffu- sion changes in radiation belt electron phase space density for the March 2013 Van Allen Probes case study, J. Geophys. Res. Space, 119, 8396-8403, doi:10.1002/2014JA020359, 2014 b.

Liu, W., Sarris, T. E., Li, X., Elkington, S. R., Ergun, R., Angelopoulos, V., Bonnell, J., and Glassmeier, K. H.: Electric and magnetic field observations of Pc4 and Pc5 pulsations in the inner magnetosphere: A statistical study, J. Geophys. Res., 114, A12206, doi:10.1029/2009JA014243, 2009.

Looper, M. D.: Response of the inner radiation belt to the violent Sun-Earth connection events of October-November 2003, Geophys. Res. Lett., 32, L03S06, doi:10.1029/2004GL021502, 2005.

Loto'aniu, T. M., Singer, H. J., Waters, C. L., Angelopoulos, V., Mann, I. R., Elkington, S. R., and Bonnell, J. W.: Relativistic electron loss due to ultralow frequency waves and enhanced outward radial diffusion, J. Geophys. Res., 115, A12245, doi:10.1029/2010JA015755, 2010.

Lyon, J., Fedder, J., and Mobarry, C.: The Lyon-Fedder-Mobarry (LFM) global MHD magnetospheric simulation code, J. Atmos. Sol.-Terr. Phy., 66, 1333-1350, doi:10.1016/j.jastp.2004.03.020, 2004.

Mann, I. R., Lee, E. A., Claudepierre, S. G., Fennell, J. F., Degeling, A., Rae, I. J., Baker, D. N., Reeves, G. D., Spence, H. E., Ozeke, L. G., Rankin, R., Milling, D. K., Kale, A., Friedel, R. H. W., and Honary, F.: Discovery of the action of a geophysical synchrotron in the Earth's Van Allen radiation belts, Nat. Commun., 4, 2795, doi:10.1038/ncomms3795, 2013.

Mauk, B. H., Fox, N. J., Kanekal, S. G., Kessel, R. L., Sibeck, D. G., and Ukhorskiy, A.: Science Objectives and Rationale for the Radiation Belt Storm Probes Mission, Space Sci. Rev., 179, 3-27, doi:10.1007/s11214-012-9908-y, 2013.

McIlwain, C. E.: Coordinates for mapping the distribution of magnetically trapped particles, J. Geophys. Res., 66, 3681-3691, doi:10.1029/JZ066i011p03681, 1961.

Merkin, V. G. and Lyon, J. G.: Effects of the low-latitude ionospheric boundary condition on the global magnetosphere, J. Geophys. Res., 115, A10202, doi:10.1029/2010JA015461, 2010.

Millan, R. M. and Thorne, R. M.: Review of radiation belt relativistic electron losses, J. Atmos. Sol.-Terr. Phy., 69, 362-377, doi:10.1016/j.jastp.2006.06.019, 2007.

Mozer, F. S., Agapitov, O., Krasnoselskikh, V., Lejosne, S., Reeves, G. D., and Roth, I.: Direct Observation of Radiation-Belt Electron Acceleration from Electron-Volt Energies to Megavolts by Nonlinear Whistlers, Phys. Rev. Lett., 113, 035001, doi:10.1103/PhysRevLett.113.035001, 2014.

Pembroke, A., Toffoletto, F., Sazykin, S., Wiltberger, M., Lyon, J., Merkin, V., and Schmitt, P.: Initial results from a dynamic coupled magnetosphere-ionosphere-ring current model, J. Geophys. Res., 117, A02211, doi:10.1029/2011JA016979, 2012.

Radoski, H. R.: A note on oscillating field lines, J. Geophys. Res., 72, 418-419, 1967.

Reeves, G. D., Spence, H. E., Henderson, M. G., Morley, S. K., Friedel, R. H. W., Funsten, H. O., Baker, D. N., Kanekal, S. G., Blake, J. B., Fennell, J. F., Claudepierre, S. G., Thorne, R. M., Turner, D. L., Kletzing, C. A., Kurth, W. S., Larsen, B. A., and Niehof, J. T.: Electron acceleration in the heart of the Van Allen radiation belts, Science, 341, 991-994, doi:10.1126/science.1237743, 2013.

Shprits, Y. Y., Thorne, R. M., Friedel, R., Reeves, G. D., Fennell, J., Baker, D. N., and Kanekal, S. G.: Outward radial dif- 
fusion driven by losses at magnetopause, J. Geophys. Res., 111, A11214, doi:10.1029/2006JA011657, 2006.

Shue, J.-H., Song, P., Russell, C. T., Steinberg, J. T., Chao, J. K., Zastenker, G., Vaisberg, O. L., Kokubun, S., Singer, H. J., Detman, T. R., and Kawano, H.: Magnetopause location under extreme solar wind conditions, J. Geophys. Res., 103, 17691, doi:10.1029/98JA01103, 1998.

Spence, H. E., Reeves, G. D., Baker, D. N., Blake, J. B., Bolton, M., Bourdarie, S., Chan, A. A., Claudepierre, S. G., Clemmons, J. H., Cravens, J. P., Elkington, S. R., Fennell, J. F., Friedel, R. H. W., Funsten, H. O., Goldstein, J., Green, J. C., Guthrie, A., Henderson, M. G., Horne, R. B., Hudson, M. K., Jahn, J.-M., Jordanova, V. K., Kanekal, S. G., Klatt, B. W., Larsen, B. A., Li, X., MacDonald, E. A., Mann, I. R., Niehof, J., O'Brien, T. P., Onsager, T. G., Salvaggio, D., Skoug, R. M., Smith, S. S., Suther, L. L., Thomsen, M. F., and Thorne, R. M.: Science Goals and Overview of the Radiation Belt Storm Probes (RBSP) Energetic Particle, Composition, and Thermal Plasma (ECT) Suite on NASA's Van Allen Probes Mission, Space Sci. Rev., 179, 311336, doi:10.1007/s11214-013-0007-5, 2013.

Thorne, R. M.: Radiation belt dynamics: The importance of wave-particle interactions, Geophys. Res. Lett., 37, doi:10.1029/2010GL044990, 2010.

Thorne, R. M., Li, W., Ni, B., Ma, Q., Bortnik, J., Chen, L., Baker, D. N., Spence, H. E., Reeves, G. D., Henderson, M. G., Kletzing, C. A., Kurth, W. S., Hospodarsky, G. B., Blake, J. B., Fennell, J. F., Claudepierre, S. G., and Kanekal, S. G.: Rapid local acceleration of relativistic radiation-belt electrons by magnetospheric chorus, Nature, 504, 411-4, doi:10.1038/nature12889, 2013.

Toffoletto, F., Sazykin, S., Spiro, R., and Wolf, R.: Inner magnetospheric modeling with the Rice Convection Model, Space Sci. Rev., 107, 175-196, doi:10.1023/A:1025532008047, 2003.
Turner, D. L., Angelopoulos, V., Li, W., Bortnik, J., Ni, B., Ma, Q., Thorne, R. M., Morley, S. K., Henderson, M. G., Reeves, G. D., Usanova, M., Mann, I. R., Claudepierre, S. G., Blake, J. B., Baker, D. N., Huang, C. L., Spence, H., Kurth, W., Kletzing, C., and Rodriguez, J. V.: Competing source and loss mechanisms due to wave-particle interactions in Earth's outer radiation belt during the 30 September to 3 October 2012 geomagnetic storm, J. Geophys. Res.-Space, 119, 1960-1979, doi:10.1002/2014JA019770, 2014.

Wiltberger, M., Wang, W., Burns, A., Solomon, S., Lyon, J., and Goodrich, C.: Initial results from the coupled magnetosphere ionosphere thermosphere model: magnetospheric and ionospheric responses, J. Atmos. Sol.-Terr. Phy., 66, 1411-1423, doi:10.1016/j.jastp.2004.03.026, 2004.

Wygant, J., Mozer, F., Temerin, M., Blake, J., Maynard, N., Singer, H., and Smiddy, M.: Large amplitude electric and magnetic field signatures in the inner magnetosphere during injection of $15 \mathrm{MeV}$ electron drift echoes, Geophys. Res. Lett., 21, 1739, doi:10.1029/94GL00375, 1994.

Wygant, J. R., Bonnell, J. W., Goetz, K., Ergun, R. E., Mozer, F. S., Bale, S. D., Ludlam, M., Turin, P., Harvey, P. R., Hochmann, R., Harps, K., Dalton, G., McCauley, J., Rachelson, W., Gordon, D., Donakowski, B., Shultz, C., Smith, C., Diaz-Aguado, M., Fischer, J., Heavner, S., Berg, P., Malsapina, D. M., Bolton, M. K., Hudson, M., Strangeway, R. J., Baker, D. N., Li, X., Albert, J., Foster, J. C., Chaston, C. C., Mann, I., Donovan, E., Cully, C. M., Cattell, C. A., Krasnoselskikh, V., Kersten, K., Brenneman, A., and Tao, J. B.: The Electric Field and Waves Instruments on the Radiation Belt Storm Probes Mission, Space Sci. Rev., 179, 183220, doi:10.1007/s11214-013-0013-7, 2013. 